\title{
Impfen in einem weltweiten Krisenfall
}

\section{Das Interview führte: Julia Rippstein}

Redaktorin SÄZ

Ende Dezember 2020 begannen die Impfungen der Schweizer Bevölkerung gegen Covid-19. Gesundheitsnotstand, Logistik, Personalrekrutierung und Mangel an Impfdosen: Die Herausforderungen sind immens und vielfältig. Hier ein Fokus auf die Impfkampagne im Kanton Waadt. Blaise Genton, Chefarzt der Poliklinik für Tropenmedizin, Reisen und Impfungen an der Unisanté und Leiter der Impfkampagne, gibt Auskunft.

\section{Blaise Genton, Sie sind der medizinische Leiter der Impfkampagne im Kanton Waadt. Was mussten Sie konkret tun, um die Impfstrategie umzusetzen?} Die Arbeit begann schon im Juni 2020; da war noch unklar, wie der Impfplan aussieht. Zu Beginn ging es darum, die verschiedenen Akteure unter der Federführung der kantonalen Gesundheitsdirektion zusammenzubringen, darunter Apotheker und Logistiker. So sollten mögliche Strategien evaluiert werden. Der Kanton ernannte einen Lenkungsausschuss und eine Task Force, die für die Umsetzung der logistischen, IT-, Kommunikations- und Personalressourcen verantwortlich ist. Als Grundlage diente uns der H1N1-Pandemieplan (Anm. d. Red.: 2009). Wir haben also nicht bei null angefangen, aber es gab doch viele Unbekannte.

\section{Vor welchen Herausforderungen standen Sie?}

Das plötzliche grüne Licht von Swissmedic zwang uns, die Impfkampagne vorzuziehen, das war sicher die erste grosse Herausforderung. Sehr schwierig an den Planungen war, dass wir die genaue Zahl der verfügbaren Dosen nicht kannten. Dieses Wissen wäre aber für die Umsetzung der Strategie entscheidend. Sie können mit einer Lieferung von 10000 Dosen nicht dasselbe planen wie mit einer von 200000 Dosen. Wir begannen mit einer nicht unerheblichen Anzahl von Dosen und hatten das Ziel, die meistgefährdeten Menschen schnell zu impfen. Eine weitere Herausforderung war, Personal für die Impfung zu finden, die Registrierung zu organisieren und die Daten der Geimpften in ein zentrales Computersystem einzugeben.

\section{Wo haben Sie all die Mitarbeitenden rekrutiert?}

In meinem 30-jährigen Berufsleben in der Schweiz habe ich zum ersten Mal gesehen, wie es ist, mit be-

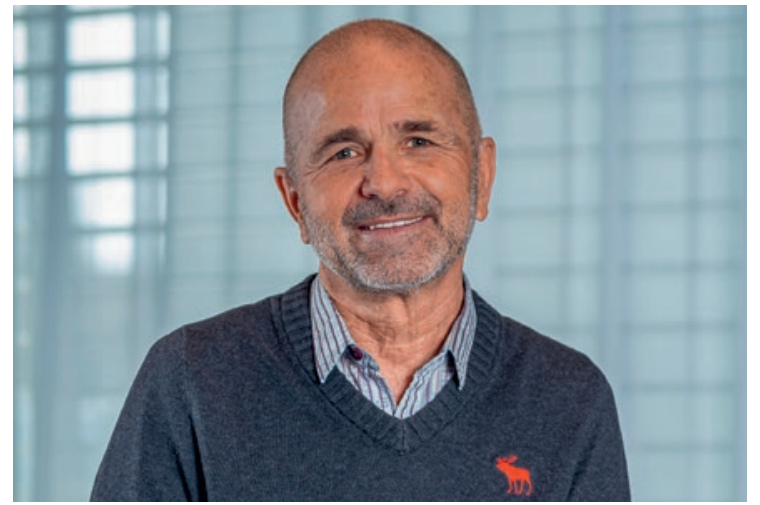

Blaise Genton: «Ärztinnen und Ärzten kommt eine wesentliche Rolle zu, denn sie kennen ihre Patientinnen und Patienten und wissen, wer die Risikopersonen sind.»

grenzten Ressourcen zu arbeiten. Wir haben pensionierte und teilzeitbeschäftigte Pflegekräfte sowie Medizin- und Pflegestudierende hinzugezogen. Der Kanton stellte uns Mitglieder des Zivilschutzes zur Verfügung. Sie kamen in die mobilen Teams, die Pflegeheime und pflegebedürftige Menschen zu Hause, aber auch Gefängnisse und Einrichtungen für Mehrfachbehinderte besuchen.

Viele dieser Personen müssen geschult werden.

Ja, das ist ein entscheidender Aspekt. Wenn Katastrophenschutzmitarbeitende, die eigentlich eine ganz andere Aufgabe haben, im Gesundheitswesen für Impfungen eingesetzt werden, müssen sie natürlich geschult werden. Auch pensionierte Mitarbeitende und Studierende müssen geschult werden. Es gibt eine hohe Fluktuation, weshalb wir laufend schulen. Und je mehr Dosen zur Verfügung stehen, desto mehr können wir natürlich impfen und desto mehr Personal brauchen wir. Dank der jetzt vorhandenen personellen 
Ressourcen und Räumlichkeiten können wir die Impfkapazität innerhalb von zehn Tagen deutlich erhöhen.

\section{Wo und wie werden die Daten der geimpften Personen erfasst?}

Das Monitoring ist ein weiterer Aspekt, der viel Zeit in Anspruch genommen hat. Als die Schweiz Pfizer/ BioNTech und später Moderna zuliess und es an der Zeit war, mit dem Impfen zu beginnen, war auf Bundesebene noch nichts für die Datenerfassung vorbereitet. Also mussten wir unser eigenes Informatiksystem entwickeln, von der Terminbuchung bis zur Ausstellung von Impfbescheinigungen. Wir haben das zum Testen von Unisanté entwickelte System übernommen und verfügen nun über Daten nahezu in Echtzeit. Ende Januar gab es dann endlich auch ein Programm des Bundes. Nun mussten wir einen Weg finden, unsere Daten in dieses zentrale System zu übertragen. Das war keine leichte Aufgabe. Viele Kantone waren in dieser Situation, und es wäre viel einfacher gewesen, von Anfang an ein einheitliches System zu entwickeln.

\section{Wie wurden die Impfzentren ausgewählt?}

In jedem der fünf Sektoren des Waadtländer Gesundheitssystems wurde ein Zentrum eröffnet, um das gesamte Gebiet abzudecken und so viele Menschen wie möglich zu erreichen. Deswegen gibt es nun ein Zentrum in der ehemaligen Bibliothek des CHUV, im Spital Rennaz-Chablais, in der Klinik La Lignière in Gland sowie in den Testzentren von Morges und Yverdon. Die Nähe zu einem Krankenhaus ist entscheidend, damit medizinisches Personal schnell mobilisiert werden kann. Mit den fünf Zentren, zusätzlich zu den mobilen Teams, können viele Menschen in kurzer Zeit geimpft werden. Wenn eine grosse Menge an Dosen geliefert wird, können wir ein zusätzliches Zentrum in Beau-

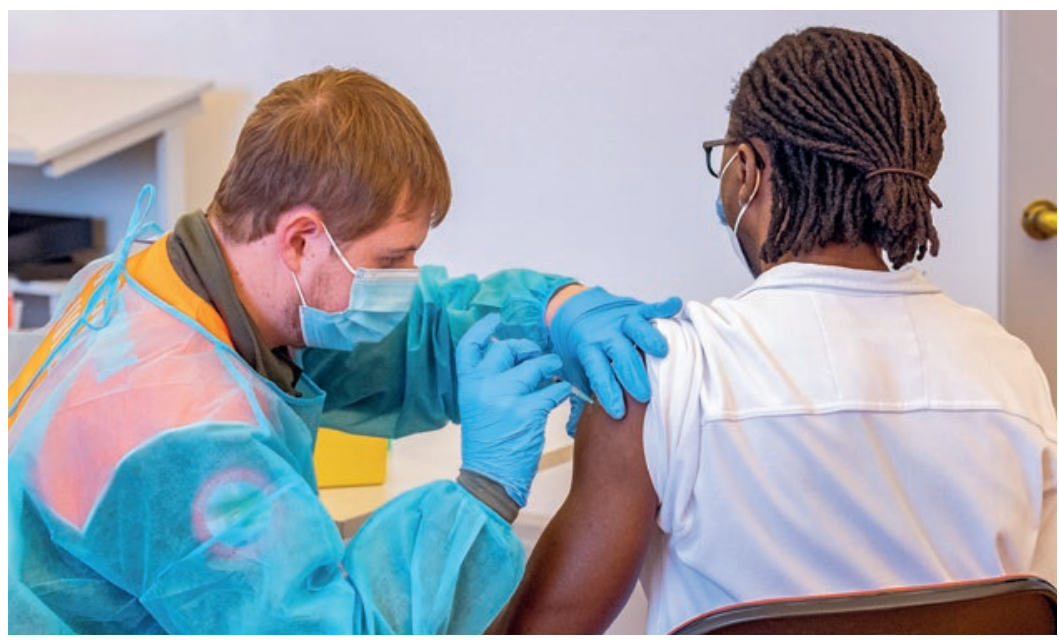

Zahlreiche Mitglieder des Zivilschutzes wurden geschult, um ab Ende Dezember 2020 für Impfungen eingesetzt zu werden. lieu, dank der Unterstützung der Klinik La Source, eröffnen und zusätzlich 3000 Personen pro Tag impfen.

\section{Der Kanton Waadt hat einen Pilotversuch durchge-} führt, um Ärztinnen und Ärzte in den Impfprozess einzubeziehen. Welche Rolle sollten sie Ihrer Meinung nach bei der Impfstrategie spielen?

Ihnen kommt eine wesentliche Rolle zu, denn sie kennen ihre Patientinnen und Patienten und wissen, wer die Risikopersonen sind. Die Bevölkerung vertraut ihnen. Wenn eine Hausärztin ihrem Patienten rät, sich impfen zu lassen, wird er diesem Rat in der Regel folgen. Die von behandelnden Ärztinnen und Ärzten vorgenommene Priorisierung ist meines Erachtens besser als die aufgrund von Bescheinigungen schwerer chronischer Erkrankungen. Im Kanton Waadt impfen 240 Ärztinnen und Ärzte bereits. Sobald wir mehr Dosen haben, werden sie voll in die Impfkampagne integriert. Ebenso werden 130 Apotheken in der Lage sein zu impfen. Wir hoffen, diese neuen Player im Laufe des Aprils einbeziehen zu können.

\section{Hausärzte und -ärztinnen haben sich jedoch beklagt,} bei der Impfstrategie aussen vor gelassen zu werden. Wurden sie zu Beginn des Prozesses übersehen?

Ich glaube nicht, dass sie übersehen wurden. Es ist kompliziert, das Impfschema in den Praxen umzusetzen. Ärztinnen und Ärzte müssen etliche halbe Tage in der Woche einplanen, um mehrere Patientinnen und Patienten hintereinander impfen zu können. Nach dem Öffnen muss eine Ampulle, d.h. sechs bzw. 10 bis 11 Dosen, innerhalb von sechs Stunden verbraucht werden. Und systematische Dateneingabe bedeutet zusätzliche Arbeit. Darüber hinaus ist die Verteilung der Ampullen an jeden behandelnden Arzt eine grosse logistische Herausforderung. Die Impfstrategie auf Ärztinnen und Ärzte zu stützen bietet sich nicht überall an. Das Wallis hat sich dafür entschieden, weil die Bevölkerung dort nicht immer in der Nähe einer grösseren Gesundheitseinrichtung lebt und sich mehr auf Hausärztinnen und -ärzte verlässt. Entscheidend sind die Geographie und die Organisation des Gesundheitssystems. Andererseits ist es in einem urbanen Kanton wie Genf sinnvoll, auf grosse Impfzentren zu setzen, die für die Mehrheit zugänglich sind.

\section{Wo werden die Dosen gelagert?}

Da der Impfstoff von Pfizer/BioNTech bei minus 70 Grad gelagert werden muss, ist ein zentraler Gefrierschrank nötig. Durch die zentrale Lagerung können wir die Dosen schnell und je nach Bedarf etwa jeden zweiten Tag an die verschiedenen Impfstellen verteilen. Die restriktiven Lagerbedingungen sind einer 


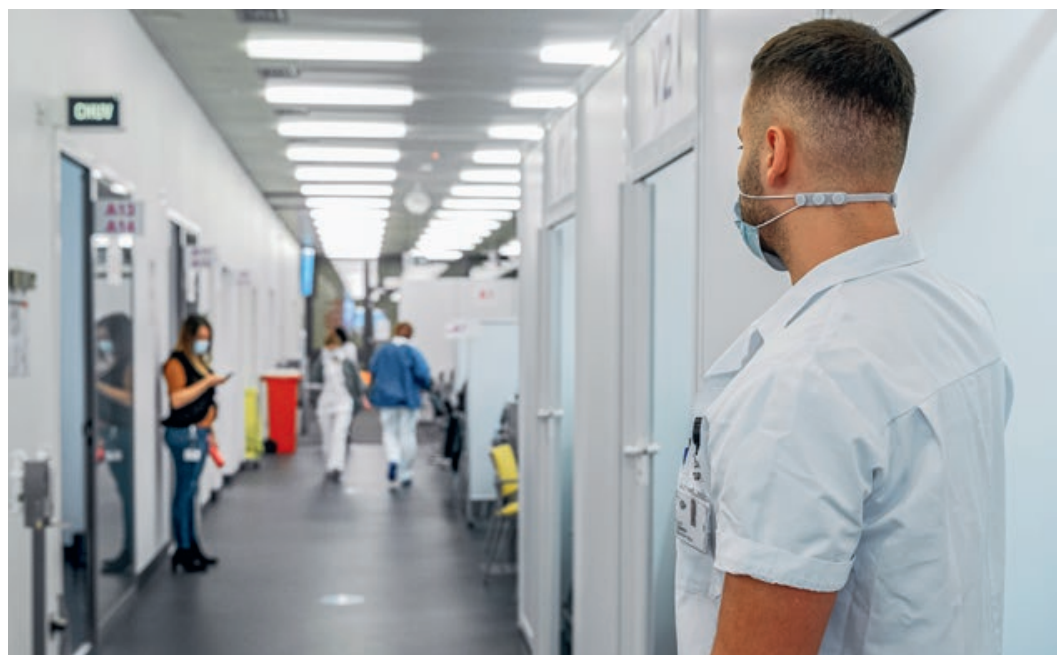

Das Impfzentrum des CHUV befindet sich in der ehemaligen Bibliothek. Die Bevölkerung der Waadt kann sich auch in Rennaz, Gland, Morges und Yverdon impfen lassen.

der Gründe, warum die behandelnden Ärztinnen und Ärzte nicht von Anfang an einbezogen wurden. Sobald die Dosen den Gefrierschrank verlassen haben, müssen sie sehr schnell verabreicht werden. Von Verschwendung kann keine Rede sein, auch wenn wir mit einem Verzug der Lieferungen konfrontiert sind und das Tempo der Impfungen erheblich verlangsamt werden musste.

\section{Lassen Sie uns über den Lieferverzug der Dosen} sprechen. Was kann in dieser Situation getan werden? Es gäbe alternative Wege, mit der Situation umzugehen, aber keiner davon wurde vom Bund beschlossen. Und es ist schwierig, von den Bundesrichtlinien abzuweichen. Im Moment lautet das Motto, so fürchte ich, «Eile mit Weile».

\section{Wie sähen diese alternativen Wege aus?}

Dem Beispiel Grossbritanniens folgend, hätte man die erste Dosis zunächst an möglichst viele Menschen verabreichen können, wissend, dass der Schutz nach $14 \mathrm{Ta}-$ gen bei fast $90 \%$ liegt, und das für wahrscheinlich drei Monate. So hätte das Intervall zwischen der ersten und der zweiten Dosis, jetzt drei bis sechs Wochen, vergrössert werden können. Dadurch könnten mehr Menschen schnell geschützt werden. Mit dieser Strategie könnten derzeit (Anm. d. Red.: 22. März) 70 Prozent der gefährdeten Personen der Gruppe 1 geimpft werden, statt etwa 50\%. Die Schweiz hat diese Strategie nicht übernommen, um sich an das Protokoll der Phase-3-Studie zu halten. Als es festgelegt wurde, war die Wirksamkeit der ersten Dosis noch nicht bekannt. Es ist schade, dass die entsprechenden Daten, die inzwischen veröffentlicht wurden, so wenig Gewicht haben. Der Bund ist sehr vorsichtig und passt sich nicht im- mer der aktuellen Lage an. Es gibt nur wenige Dosen, während der Gesundheitsnotstand anhält. Aber die Strategie wird nicht überprüft.

\section{Aber wir können doch froh sein, dass die Schweiz vorsichtig ist, oder?}

Dahinter verbirgt sich die verzwackte Herausforderung "Individualschutz versus Bevölkerungsschutz». Indem wir einen kleinen Teil der Bevölkerung sehr gut schützen, wird der Einfluss auf die Zahl der schweren Verläufe und der Hospitalisierungen und auf die Verbreitung des Virus geringer sein. Wenn das Virus zirkuliert, wird es unweigerlich mutieren, und neue Varianten werden erscheinen.

\section{Was tut der Kanton Waadt, um Dosen zu sparen?} Wir haben die Anzahl Impfberechtigter beim Gesundheitspersonal reduziert. Nur Personen, die auf der Intensivstation, in der Notaufnahme und in Prüfeinrichtungen arbeiten, werden geimpft. Wir haben auch entschieden, Personen keine Priorität einzuräumen, die bereits an Covid-19 erkrankt waren. Auch nicht, wenn dies mehr als drei Monate zurückliegt, denn die Rate der Reinfektion ist sehr gering. Dies führte zu kritischen Fragen aus anderen Kantonen und vom Bund. Es ist eine Entscheidung zwischen öffentlicher Gesundheit und dem totalen Schutz Einzelner.

\section{Halten Sie das Ziel, bis Ende Juni alle Impfwilligen geimpft zu haben, heute für realistisch?}

Ende Juli scheint mir realistischer. Im Kanton Waadt war das Ziel, bis Ende Februar alle gefährdeten Personen der Gruppe 1 zu impfen, und zwar mit einer Rate von 4000 Personen pro Tag. Wegen der Verspätung der Dosen und ohne einen anderen zugelassenen Impfstoff wird dies aber wahrscheinlich erst Ende März der Fall sein. Wir sind derzeit bei einer Rate von 2000 bis 3000 Dosen pro Tag, anstatt von möglichen 10000.

\section{Wann kommen die nächsten Dosen?}

Wir erhalten regelmässig neue Dosen, etwa zweimal pro Woche. Unsere Planungen gehen über das gesamte Jahr 2021, entsprechend der Mengen, die uns zugesagt wurden. Wenn wir vom Bund die Bestätigung haben, dass Dosen ankommen, dann kommen sie bereits am nächsten Tag. Solch knappe Fristen erfordern eine sehr genaue Planung. Das grosse Fragezeichen im Moment ist, wann andere Impfstoffe zugelassen werden, wie z.B. der Impfstoff von AstraZeneca. Wenn er zugelassen wird, sollten wir die geplanten Tausenden von Dosen erhalten und sie sehr schnell verteilen können.

\section{Bildnachweise}

(C) Porträt: Unisanté

(c) Andere: CHUV 2021 